\title{
AN ANALYTICAL FRAMEWORK FOR NUMERICAL HOMOGENIZATION. PART II: WINDOWING AND OVERSAMPLING*
}

\author{
ANTOINE GLORIA ${ }^{\dagger}$
}

\begin{abstract}
In a recent paper [Multiscale Model. Simul., 5 (2006), pp. 996-1043], the author has introduced an analytical framework to study the convergence properties of some numerical homogenization methods for elliptic problems. In the applications however, these methods are coupled with windowing or oversampling techniques. In the present work, the author addresses this issue within the latter framework and proves the convergence of the methods with windowing, for convex and quasiconvex energies, in the context of general heterogeneities. This analysis provides us with an interesting variational interpretation of the Petrov-Galerkin formulation of the nonconforming multiscale finite element method for periodic problems.
\end{abstract}

Key words. homogenization, finite element, windowing, oversampling

AMS subject classifications. 77Q15, 74S05, 35J60

DOI. $10.1137 / 070683143$

1. Introduction. The goal of this paper is to continue the analysis of multiscale methods for the numerical homogenization of elliptic equations initiated in [18] and [17], where the convergence and the numerical analysis of some numerical homogenization methods are addressed under quite general hypotheses on the heterogeneities (general spatial dependence of the operator) and on the nature of the operator (convex or quasiconvex associated energy density). In practice, however, these methods are usually combined with more sophisticated techniques such as windowing and oversampling. Windowing techniques basically amount to imposing boundary conditions further from the region of interest to minimize their effects. It gives rise to oversampling techniques for the multiscale finite element method (MsFEM) and is also used in the heterogeneous multiscale method (HMM). It is implicitly used or referred to in [2], [19], [20], [9], [11], [13], and theoretically analyzed in [21], [10], and $[8]$ in the linear and periodic or stochastic cases, for which error estimates are also provided ([10], [21] for the periodic case and [8] for the stochastic case). In the present work, we focus on proving the convergence of some numerical homogenization methods with windowing under the same general hypotheses as in [17], concerning both the approximation of the homogenized solution and the corrector whenever the notion is well established. The paper is organized as follows. In the first section, we very briefly recall the context, the main results of [17], and two numerical methods. Then we discuss the issue of windowing in the periodic case, before addressing it in a more general setting in section 4 . We also give a variational interpretation of the nonconforming Petrov-Galerkin formulation of the multiscale finite element method that allows us to prove its convergence in a general setting. Some arguments and proofs are sketched only whenever they are mainly based on the corresponding ones

* Received by the editors February 20, 2007; accepted for publication (in revised form) September 19, 2007; published electronically April 30, 2008.

http://www.siam.org/journals/mms/7-1/68314.html

${ }^{\dagger}$ CERMICS, Ecole Nationale des Ponts et Chaussées, 6 et 8 avenue Blaise Pascal, Cité Descartes, 77455 Marne-la-Vallée Cedex 2, France, and Project MICMAC, INRIA, Rocquencourt, 78513 Le Chesnay Cedex, France (gloria@cermics.enpc.fr). 
in [17]. We refer the reader to [17, sect. 1] and the references therein for further details on notation and useful results that may not be extensively recalled in section 2 .

2. Numerical homogenization methods. Let us recall the analytical framework introduced in [17] and two numerical methods to which the analysis applies: the HMM and the MsFEM.

2.1. Main notation. Let us introduce the major notation used in what follows. Given a function $v$ defined on an open set $\mathcal{O}$, we set

$$
\langle v\rangle_{\mathcal{O}}=\frac{1}{|\mathcal{O}|} \int_{\mathcal{O}} v
$$

For a metric space $(V, d)$, we say that a functional $F_{\epsilon}: V \rightarrow \mathbb{R} \Gamma(d)$-converges to a functional $F: V \rightarrow \mathbb{R}$ if for all $v \in V$

$$
F(v)=\inf _{\left\{v_{\epsilon}\right\} \in V^{\mathbb{N}}, d\left(v_{\epsilon}-v\right) \rightarrow 0}\left\{\liminf _{\epsilon \rightarrow 0} F_{\epsilon}\left(v_{\epsilon}\right)\right\}=\inf _{\left\{v_{\epsilon}\right\} \in V^{\mathbb{N}}, d\left(v_{\epsilon}-v\right) \rightarrow 0}\left\{\limsup _{\epsilon \rightarrow 0} F_{\epsilon}\left(v_{\epsilon}\right)\right\},
$$

and we denote it by $F=\Gamma(d)-\lim _{\epsilon \rightarrow 0} F_{\epsilon}$. We refer the reader to [17, sect. 1] and the references therein for some useful properties of $\Gamma$-convergence. The metric space we will use to state the $\Gamma$-convergence results are either Sobolev spaces or Lebesgue spaces. In particular we will set

$$
W_{\#}^{1, p}(Q)=\left\{v_{\mid Q}, v \in W_{l o c}^{1, p}\left(\mathbb{R}^{n}\right), v(x)=v(x+Q) \text { for almost every } x \in \mathbb{R}^{n}\right\},
$$

where $Q$ is a hypercube.

We also adopt generic conventions for the following symbols:

- $\Omega$ : open bounded domain of $\mathbb{R}^{n}$;

- $x$ : generic point in $\Omega$ (or more generally in $\mathbb{R}^{n}$ );

- $C(x, \eta)$ : cube of length $\eta>0$ centered at point $x$;

- $y$ : generic point in $C(x, \eta)$;

- $u$ : (with various indices) function in $W^{1, p}(\Omega)$;

- $v$ : (with various indices) function in $W^{1, p}(C(x, \eta))$ for some $x \in \Omega$ and $\eta>0$.

2.2. Minimization problem. In what follows, we consider minimization problems, or the associated Euler-Lagrange equations, whenever the two approaches are equivalent, e.g., for monotone operators. The problem under investigation is

$$
\inf \left\{\int_{\Omega} W_{\epsilon}(x, \nabla u) d x, u \in W^{1, p}\left(\Omega, \mathbb{R}^{d}\right)+B C\right\},
$$

where $W_{\epsilon}$ is a family of energy densities and $B C$ denotes classical boundary conditions (let us say Dirichlet or mixed Dirichlet-Neumann boundary conditions; see [17, p. 999]) in weak form.

Denoting by $I_{\epsilon}: W^{1, p}(\Omega) \rightarrow \mathbb{R}, u \mapsto I_{\epsilon}(u)=\int_{\Omega} W_{\epsilon}(x, \nabla u) d x$, we want to study the behavior of $I_{\epsilon}$ when $\epsilon$ vanishes and to numerically approximate the minimizers of $I_{\epsilon}$ on sets of prescribed boundary conditions.

Under the following sets of hypotheses on $W_{\epsilon}$, both issues can be answered positively.

The convex case.

- H1: $W_{\epsilon}$ is a Carathéodory function;

- H2: for almost every $x \in \mathbb{R}^{d \times n}, W_{\epsilon}(x, \cdot)$ is convex on $\mathbb{R}^{d \times n}$; 
- H3: there exist $0<c \leq C$ and $p \geq 1$ such that

$$
c|\xi|^{p}-1 \leq W_{\epsilon}(x, \xi) \leq C\left(1+|\xi|^{p}\right)
$$

for almost all $x \in \mathbb{R}^{n}$ and for all $\xi \in \mathbb{R}^{n}$.

The quasiconvex case: $n>1$ and $d>1$.

- $\mathrm{H} 1$;

- H4: for almost every $x \in \mathbb{R}^{n}, W_{\epsilon}(x, \cdot)$ is quasiconvex on $\mathbb{R}^{d \times n}$;

- $\mathrm{H} 3$.

Assumption $\mathrm{H} 4$ generalizes assumption H2. An energy density satisfying H1, H4, and $\mathrm{H} 3$ will be referred to as a standard energy density. The direct method of the calculus of variations shows that the minimization problem (2.1) has at least one solution $u_{\epsilon} \in W^{1, p}\left(\Omega, \mathbb{R}^{d}\right)$.

In addition, there exist a standard energy density $W_{\text {hom }}$ and the associated energy functional $I_{\text {hom }}: W^{1, p}(\Omega) \rightarrow \mathbb{R}, u \mapsto I_{h o m}(u)=\int_{\Omega} W_{h o m}(x, \nabla u) d x$, such that, up to extraction, $I_{h o m}=\Gamma\left(L^{p}\right)-\lim _{\epsilon \rightarrow 0} I_{\epsilon}$. For every sequence of minimizers $u_{\epsilon}$, there exists a minimizer $u_{\text {hom }}$ of $I_{\text {hom }}$ on the same set of prescribed boundary conditions such that $u_{\epsilon} \rightarrow u_{\text {hom }}$ in $W^{1, p}(\Omega)$. In what follows we will consider $\Gamma$-converging energies, without loss of generality up to extraction.

The aim of the following section is to recall the definition of an averaged energy density $I_{\eta, \epsilon}$ that approximates $I_{h o m}$ and $u_{h o m}$ in the sense of $\Gamma$-convergence.

2.3. Averaged energy densities. For convex standard energy densities $W_{\epsilon}$, we set the following.

Definition 1. For any $\eta>0$, denoting by $B(x, \eta)$ the ball of radius $\eta$ centered at point $x \in \mathbb{R}^{n}$, we define the energy density

$$
W_{\eta, \epsilon}(x, \xi)=\inf \left\{\left\langle W_{\epsilon}(\cdot, \xi+\nabla v(\cdot))\right\rangle_{B(x, \eta)} \mid v \in W^{1, p}(B(x, \eta)),\langle\nabla v\rangle_{B(x, \eta)}=0\right\}
$$

from $\mathbb{R}^{n} \times \mathbb{R}^{d \times n}$ to $\mathbb{R}$ and the associated energy functional

$$
I_{\eta, \epsilon}(u)=\int_{\Omega} W_{\eta, \epsilon}(x, \nabla u) \quad \text { for all } u \in W^{1, p}(\Omega)
$$

whereas for quasiconvex standard energy densities, we set the following.

Definition 2. For $\eta>0$, let us denote by $C(x, \eta)$ the hypercube of $\mathbb{R}^{n}$ centered in $x \in \mathbb{R}^{n}$ and of length $\eta$. We then define the averaged energy density by

$$
\mathcal{W}_{\eta, \epsilon}(x, \xi)=\inf \left\{\left\langle W_{\epsilon}(\cdot, \xi+\nabla v(\cdot))\right\rangle_{C(x, \eta)} \mid v \in W_{\#}^{1, p}\left(C(x, \eta), \mathbb{R}^{d}\right)\right\}
$$

from $\mathbb{R}^{n} \times \mathbb{R}^{d \times n}$ to $\mathbb{R}$ and the energy functional associated with its quasiconvex envelope $Q \mathcal{W}_{\eta, \epsilon}$

$$
I_{\eta, \epsilon}(u)=\int_{\Omega} Q \mathcal{W}_{\eta, \epsilon}(x, \nabla u) \quad \text { for all } u \in W^{1, p}\left(\Omega, \mathbb{R}^{d}\right)
$$

We then have the two following convergence theorems.

THEOREM 1. Let $W_{\epsilon}$ satisfy $\mathrm{H} 1, \mathrm{H} 2$ (strictly), and $\mathrm{H} 3$ uniformly for $p>1$; then the energy densities $W_{\eta, \epsilon}$ also satisfy $\mathrm{H} 1, \mathrm{H} 2$ (strictly), and $\mathrm{H} 3$, and the energy $I_{\eta, \epsilon} \Gamma\left(L^{p}\right)$ - and $\Gamma\left(W^{1, p}\right)$-converges to $I_{h o m}$ as $\epsilon$ and $\eta$ go to 0 . Therefore, the unique sequence $u_{\eta, \epsilon}$ of minimizers of $I_{\eta, \epsilon}$ on $W^{1, p}(\Omega, \mathbb{R})+B C$ strongly converges in $W^{1, p}\left(\Omega, \mathbb{R}^{n}\right)$ to the unique minimizer $u_{\text {hom }}$ of $I_{\text {hom }}$ on $W^{1, p}(\Omega, \mathbb{R})+B C$.

Copyright $\odot$ by SIAM. Unauthorized reproduction of this article is prohibited. 
TheOREm 2. Let $W_{\epsilon}$ satisfy $\mathrm{H} 1, \mathrm{H} 4$, and $\mathrm{H} 3$ uniformly for $p>1$; then the energy densities $Q \mathcal{W}_{\eta, \epsilon}$ are standard energy densities, and $I_{\eta, \epsilon} \Gamma\left(L^{p}\right)$ - and $\Gamma\left(W^{1, p}\right)$-converges to $I_{\text {hom }}$ as $\epsilon$ and $\eta$ go to 0 . Therefore, for any sequence $u_{\eta, \epsilon}$ of minimizers of $I_{\eta, \epsilon}$ on $W^{1, p}\left(\Omega, \mathbb{R}^{d}\right)+B C$, there exists a minimizer $u_{\text {hom }}$ of $I_{h o m}$ on $W^{1, p}\left(\Omega, \mathbb{R}^{d}\right)+B C$ such that

$$
\lim _{\eta \rightarrow 0} \lim _{\epsilon \rightarrow 0} u_{\eta, \epsilon}=u_{\text {hom }} \quad \text { weakly in } W^{1, p}\left(\Omega, \mathbb{R}^{d}\right)
$$

up to extraction.

REMARK 1. It may be stressed that the trial spaces $\left\{v \in W^{1, p}\left(B(x, \eta), \mathbb{R}^{d}\right)\right.$, $\langle\nabla v\rangle=0\}$, and $W_{\#}^{1, p}\left(C(x, \eta), \mathbb{R}^{d}\right)$ can be replaced, respectively, by $W_{0}^{1, p}\left(B(x, \eta), \mathbb{R}^{d}\right)$ in $(2.2)$ and $W_{0}^{1, p}\left(C(x, \eta), \mathbb{R}^{d}\right)$ in (2.3) without changing the convergence results of Theorems 1 and 2.

REMARK 2. The order of the limits in (2.4) is important and cannot be changed in general.

2.4. Numerical corrector and fine scale features. The approximation of the homogenized solution $u_{\text {hom }}$ in $W^{1, p}(\Omega)$ is not enough to approximate $u_{\epsilon}$ in $W^{1, p}(\Omega)$ since $u_{\epsilon}$ converges only weakly to $u_{\text {hom }}$ in $W^{1, p}(\Omega)$. To this aim, numerical correctors have been widely introduced and used to approximate $\nabla u_{\epsilon}$ in $L^{p}(\Omega)$, which describes the fine scale features of the solution. Their convergence properties have been analyzed for general heterogeneities and monotone operators in [17].

DeFINITION 3. Let $\left\{Q_{H, i}\right\}_{i \in \llbracket 1, I_{H} \rrbracket}$ be a partition of $\Omega$ in disjoint subdomains of diameter of order $H$. Keeping the notation of Theorem 1, for all $i$, we define the numerical correctors $v_{\eta, \epsilon}^{H, i}$ for a strictly convex energy density as the unique minimizers (up to a constant) of

$$
\inf \left\{\int_{Q_{H, i}} W_{\epsilon}(x, \nabla v) \mid v \in W^{1, p}\left(Q_{H, i}\right),\langle\nabla v\rangle_{Q_{H, i}}=\left\langle\nabla u_{\eta, \epsilon}\right\rangle_{Q_{H, i}}\right\} .
$$

The following convergence result holds [17, Thm. 2].

TheOrem 3. In addition to $\mathrm{H} 1, \mathrm{H} 2$, and $\mathrm{H} 3$, let us assume that $p \geq 2$, that $W_{\epsilon}(x, \cdot)$ is continuously differentiable for almost all $x \in \Omega$ and $a_{\epsilon}(\cdot, 0)=\frac{\partial \bar{W}_{\epsilon}}{\partial \xi}(\cdot, 0)$ is bounded, and that the following monotonicity and continuity properties hold:

$$
\begin{aligned}
& \exists 0 \leq \alpha \leq p-1, C>0 \quad \mid \quad \text { for almost all } x \in \mathbb{R}^{n}, \text { for all } \xi_{1}, \xi_{2} \in \mathbb{R}^{n}, \\
& \quad\left|a_{\epsilon}\left(x, \xi_{1}\right)-a_{\epsilon}\left(x, \xi_{2}\right)\right| \leq C\left(1+\left|\xi_{1}\right|+\left|\xi_{2}\right|\right)^{p-1-\alpha}\left|\xi_{1}-\xi_{2}\right|^{\alpha}, \\
& \exists 2 \leq \beta<+\infty, c>0 \quad \mid \quad \text { for almost all } x \in \mathbb{R}^{n}, \text { for all } \xi_{1}, \xi_{2} \in \mathbb{R}^{n}, \\
& \quad\left(a_{\epsilon}\left(x, \xi_{1}\right)-a_{\epsilon}\left(x, \xi_{2}\right), \xi_{1}-\xi_{2}\right) \geq c\left(1+\left|\xi_{1}\right|+\left|\xi_{2}\right|\right)^{p-\beta}\left|\xi_{1}-\xi_{2}\right|^{\beta} .
\end{aligned}
$$

Then, denoting by $u_{\epsilon}$ the unique minimizer of $I_{\epsilon}$ on $W^{1, p}(\Omega)+B C$, we have

$$
\lim _{\eta, H \rightarrow 0} \lim _{\epsilon \rightarrow 0}\left\|\nabla u_{\epsilon}-\sum_{i=1}^{I_{H}} \nabla v_{\eta, \epsilon}^{H, i} 1_{Q_{H, i}}\right\|_{L^{p}(\Omega)}=0 .
$$

Let us briefly discuss the link between the original Tartar correctors and the numerical correctors of Definition 3. We first recall the definition of Tartar's correctors [24] in the linear case and make some comments about the numerical interest of such a result.

Copyright $@$ by SIAM. Unauthorized reproduction of this article is prohibited. 
Let $A_{\text {hom }} \in L^{\infty}\left(\Omega, \mathcal{M}_{n}(\mathbb{R})\right)$ be the homogenized matrix of an $H$-converging sequence $A_{\epsilon}$ (see [24], e.g.) and $u_{\text {hom }}$ be the solution of the homogenized problem $\inf \left\{\int_{\Omega} \nabla u \cdot A_{\text {hom }} \nabla u-f u, u \in H^{1}(\Omega)+B C\right\}$. The convergence of $u_{\epsilon}$ to $u_{\text {hom }}$ is weak only in $H^{1}(\Omega)$. The corrector matrices are designed to approximate the gradient of $u_{\epsilon}$ by a function depending linearly on $\nabla u_{\text {hom }}$. Given compactly supported subsets $\omega \subset \subset \omega_{1} \subset \subset \Omega$, a corrector matrix $P_{\epsilon} \in H^{1}(\omega)^{n}$ is defined by its entries $P_{\epsilon} \cdot e_{j}=P_{\epsilon}^{j} \in H^{1}(\omega)$, where $P_{\epsilon}^{j}$ is the restriction on $\omega$ of the unique solution of

$$
\inf \left\{\int_{\omega_{1}} \nabla v_{\epsilon} \cdot A_{\epsilon} \nabla v_{\epsilon}-e_{j} \cdot A_{h o m} \nabla v_{\epsilon}, v_{\epsilon}(y)=e_{j} \cdot y+w_{\epsilon}, w_{\epsilon} \in H_{0}^{1}\left(\omega_{1}\right)\right\}
$$

for $e_{j}$ describing the canonical basis of $\mathbb{R}^{n}$. A corrector for $u_{\epsilon}$ is then given on $\omega$ by

$$
C_{\epsilon}=\sum_{j}\left(\nabla u_{h o m} \cdot e_{j}\right) \nabla P_{\epsilon}^{j}
$$

As a sum of products of two $L^{2}$-functions, $C_{\epsilon} \in L^{1}(\omega)^{n}$, and we have [24, Thm. 3 p. 39])

$$
\lim _{\epsilon \rightarrow 0}\left\|C_{\epsilon}-\nabla u_{\epsilon}\right\|_{L^{1}(\omega)}=0 .
$$

It is worth noticing that correctors are not gradient fields in general. In addition, correctors are not unique.

From a computational point of view, Tartar's correctors are too abstract since the precise knowledge of $A_{h o m}$ is required to calculate the correctors, whereas $A_{h o m}$ is in principle unknown. However, if $A_{\text {hom }}$ is constant, then (2.9) turns out to be solvable in practice (the term depending on $A_{h o m}$ is constant in the energy). As pointed out by Allaire and Brizzi in [2], the simplest approximation of $A_{h o m}$ is the piecewise constant approximation.

The numerical corrector amounts to taking $\omega=\omega_{1}=Q_{H, i}$ and approximating $A_{\text {hom }}$ by $\left\langle A_{\eta, \epsilon}\right\rangle_{Q_{H, i}}$. It should be noticed that $\omega$ is not compactly supported in $\omega_{1}$. In addition to the convergence in $\epsilon$ (and $\eta$ ), there is an error linked to $H$ and the piecewise constant approximation of $A_{\text {hom }}$. Up to an error which depends on $H$, the numerical corrector may be interpreted as an approximation of some Tartar corrector on $Q_{H, i}$. In addition, Theorem 3 shows that the convergence of the numerical corrector holds in $L^{2}(\Omega)$. Imposing $\omega \subset \subset \omega_{1}$ in the definition of a numerical corrector is a way to introduce windowing, as will be seen in section 4 .

2.5. HMM. The application of the HMM to elliptic problems introduced in [7] can be interpreted as the minimization of a discretization of $I_{\eta, \epsilon}$ plus a lower order term $f \in L^{p^{\prime}}(\Omega)$ on a finite element basis, which reads

$$
\inf \left\{\sum_{i=1}^{I_{H}} \sum_{j=1}^{N_{G P}} q_{j}\left(W_{\eta, \epsilon}\left(x_{i j}, \nabla u_{H}\left(x_{i j}\right)\right)-f\left(x_{i j}\right) u_{H}\left(x_{i j}\right)\right), u_{H} \in V_{H}+B C\right\},
$$

where $I_{H}$ is the number of mesh elements, $N_{G P}$ is the number of Gauss points per element, $x_{i j}$ are the Gauss points, $q_{j}$ are the weights, and $V_{H}$ is a FE space. Then the computation of the FE minimizer of (2.10) requires only evaluations of derivatives of $W_{\eta, \epsilon}\left(x_{i j}, \xi\right)$ for particular $\xi$ at Gauss points $x_{i j}$. We refer the reader to [7], [17] for details on the method and its analysis. 
2.6. MsFEM. The MsFEM may also be interpreted as the minimization of a (different) discretization of $I_{\eta, \epsilon}$ on a $P 1$-FE space $V_{H}$ associated with a triangulation $\left\{Q_{H, i}\right\}_{i}$ of $\Omega$, namely

$$
\begin{aligned}
\inf \left\{\sum_{i=1}^{I_{H}}\left|Q_{H, i}\right| W_{H, \epsilon}^{M s F E M}\left(x_{i}, \nabla u_{H}\left(x_{i}\right)\right)\right. & \\
& \left.-\sum_{i=1}^{I_{H}} \sum_{j=1}^{N_{G P}} q_{j} f\left(x_{i j}\right) u_{H}\left(x_{i j}\right), u_{H} \in V_{H}+B C\right\},
\end{aligned}
$$

where $x_{i}$ typically denotes the center of $Q_{H, i}$, the second term of the energy has been integrated by a quadrature rule on Gauss points $x_{i j}$, and

$$
W_{H, \epsilon}^{M s F E M}\left(x_{i}, \xi\right)=\inf \left\{\frac{1}{\left|Q_{H, i}\right|} \int_{Q_{H, i}} W_{\epsilon}(y, \nabla v) \mid v(y)=\xi \cdot y \text { on } \partial Q_{H, i}\right\},
$$

which is a particular energy density of type $W_{\eta, \epsilon}$.

In the analysis of the MsFEM in [17, sect. 3.2], we have extensively used the following rewriting of the problem:

$$
\inf \left\{\int_{\Omega} W_{H, \epsilon}^{M s F E M}\left(x, \nabla u_{H}\right)-f u_{H} \mid u_{H} \in V_{H}+B C\right\},
$$

extending the definition of $W_{H, \epsilon}^{M S F E M}$ on $\Omega$ by

$$
W_{H, \epsilon}^{M s F E M}(x, \xi)=\sum_{i} W_{H, \epsilon}^{M s F E M}\left(x_{i}, \xi\right) 1_{Q_{H, i}}(x),
$$

since $\nabla u_{H}$ is constant on each $Q_{H, i}$. With this formulation, estimates on the energy are easy to obtain due to the inclusion $V_{H} \subset W^{1, p}(\Omega)$.

To relate this formulation with the original formulation of the MsFEM, it is enough to notice that for any $u_{H} \in V_{H}$ one can define the restriction of the associated multiscale finite element $u_{H, \epsilon}^{M s F E M}$ (at least in the monotone case) on each mesh element $Q_{H, i}$ as the unique solution of

$$
\inf \left\{\frac{1}{\left|Q_{H, i}\right|} \int_{Q_{H, i}} W_{\epsilon}(y, \nabla v) \mid v(y)=\nabla u_{H} \cdot y \text { on } \partial Q_{H, i}\right\} .
$$

We then have

$$
\int_{\Omega} W_{H, \epsilon}^{M s F E M}\left(x, \nabla u_{H}\right)=\int_{\Omega} W_{\epsilon}\left(x, \nabla u_{H, \epsilon}^{M s F E M}\right) .
$$

Other numerical methods can be recast within this analytical framework, such as the residual-free bubbles finite element method introduced in [26], where the duality of points of view $\left(W_{H, \epsilon}^{M s F E M}, V_{H}\right)$ and $\left(W_{\epsilon},\left\{u_{H, \epsilon}^{M s F E M}\right\}\right)$ is pointed out.

3. Windowing in the periodic case. As a matter of fact, windowing is often used even if not always mentioned. The present terminology is borrowed from the mechanical community. Applied to the MsFEM, windowing is referred to as oversampling. Combined with the HMM, it has no particular name, and we will make use of the mechanical terminology. 
3.1. Setting of the problem. Since the homogenized equation is explicitly known when $W_{\epsilon}(\cdot, \cdot)=W\left(\frac{\dot{\epsilon}}{\epsilon}, \cdot\right)$ and $W$ is 1-periodic in space, the application of the MsFEM or the HMM strategies to this case allows us to perform a numerical analysis of the methods.

Doing so ([21] for the MsFEM and [8, eq. (1.8a)] for the HMM, e.g.), the error between the numerical approximation and the solution of the homogenized problem is proved to exhibit some term called the cell resonance and boundary layer error. This error is linked to two phenomena:

- when $C(x, \eta)$ is not a multiple of the periodic cell on the one hand (cell resonance), and

- when linear Dirichlet boundary conditions are used on the other hand (the cell problem in periodic homogenization is completed by periodic boundary conditions).

These phenomena are the sources of a boundary layer, which gives the following error in the linear case between the solutions of the homogenized and averaged problems:

$$
\left\|u_{h o m}-u_{\eta, \epsilon}\right\|_{H^{1}(\Omega)} \leq C \frac{\epsilon}{\eta}
$$

(the same estimate holds for the error between the homogenized and averaged coefficients of the linear operator). For the error on the correctors, it yields

$$
\left\|C_{\epsilon}-\sum_{i=1}^{I_{H}} \nabla v_{\eta, \epsilon}^{H, i} 1_{Q_{H, i}}\right\|_{L^{2}(\Omega)} \leq C \sqrt{\frac{\epsilon}{\eta}},
$$

where $C_{\epsilon}$ now denotes the two-scale corrector for periodic homogenization (see $[1$, Thm. 9.9] for a definition of the corrector and [10], [21] for the error estimate). We refer the reader to [17, Prop. 1] for the corresponding estimates in the monotone case. The aim of windowing is twofold: improve the convergence rate, and if not, at least, improve the prefactor, by reducing both sources of error.

In the linear periodic case, windowing restores a convergence of order $\frac{\epsilon}{\eta}$ for the $L^{2}(\Omega)$-norm of the corrector and reduces the prefactor multiplying the error of order $\frac{\epsilon}{\eta}$ for the homogenized coefficients ([8] and [19], e.g.). The strategy consists in introducing bigger volume elements $C(x, \eta+\zeta$ ), where $\zeta=\zeta(\epsilon, \eta)>0$ (hence the term windowing), and using the information only on $C(x, \eta)$ to avoid the boundary layer of presumed order $\zeta$. The application of windowing is different for the MsFEM and the HMM. In particular, for the MsFEM, several choices (mainly depending on the relative weights for the construction of the MsFEM basis; see Remark 7 for one variant) are possible. One of them has been analyzed in great detail in [21] in the linear periodic case. The mathematical formulation of windowing is introduced in the following section.

3.2. Mathematical formulation. Let $\zeta=\zeta(\epsilon, \eta) \in \mathbb{R}_{+}$. We define a "windowed" energy density

$$
W_{\eta, \epsilon, \zeta}^{w i n}(x, \xi)=\left\langle W_{\epsilon}\left(y, \xi+\nabla v_{\eta, \epsilon, \zeta}^{w i n}(y)\right)\right\rangle_{C(x, \eta)},
$$

where $v_{\eta, \epsilon, \zeta}^{w i n}$ is the restriction on $C(x, \eta)$ of the solution $\tilde{v}_{\eta, \epsilon, \zeta}^{w i n}$ of the following minimization problem posed on $C(x, \eta+\zeta)$ :

$$
\inf \left\{\left\langle W_{\epsilon}(\cdot, \xi+\nabla v(\cdot))\right\rangle_{C(x, \eta+\zeta)} \mid v \in W_{0}^{1, p}(C(x, \eta+\zeta))\right\} .
$$


We can naturally extend this definition to balls $B(x, \eta)$ and other boundary conditions $(\langle\nabla v\rangle=\xi$, periodic boundary conditions). Since the arguments and results are the same, we will focus on Dirichlet boundary conditions in what follows.

For the MsFEM with oversampling, we will adopt the following notation:

$$
\int_{\Omega} W_{H, \epsilon, \text { over }}^{M s F E M}\left(x, \nabla w_{H}\right)=\sum_{i} \int_{Q_{H, i}} W_{\epsilon}\left(x, \nabla w_{H, \epsilon, \text { over } \mid Q_{H, i}}^{M s F E M}\right),
$$

where $w_{H, \epsilon, \text { over }}^{M \text { sFEM }} \mid Q_{H, i}$ is the restriction on $Q_{H, i}$ of a solution of

$$
\inf \left\{\int_{Q_{H+\zeta, i}} W_{\epsilon}(y, \nabla v) \mid v(y)=\nabla w_{H} \cdot y \text { on } \partial Q_{H+\zeta, i}\right\}
$$

and $Q_{H+\zeta, i}$ is an extension of $Q_{H, i}$ such that $\zeta \leq d\left(\partial Q_{H, i}, \partial Q_{H+\zeta, i}\right) \leq 2 \zeta$. In what follows, we will also make use of the oscillating part of $\nabla w_{H, \epsilon, \text { over }}^{M s F E M Q_{H, i}}$, namely

$$
\nabla w_{\epsilon, \text { over }}^{H, i}(y)=\left.\nabla w_{H, \epsilon, \text { over }}^{M \text { SFEM }}\right|_{Q_{H, i}}-\left\langle\nabla w_{H, \epsilon, \text { over }}^{M \text { sFEM }}{ }_{\mid Q_{H, i}}\right\rangle_{\mid Q_{H, i}} .
$$

We will not make a full error analysis of the cancellations that occur due to windowing, and we refer the reader to [21] for a deep analysis in the linear case. We will, however, make two remarks that may explain why numerical errors are reduced by the use of windowing methods. The aim of the present work is to prove the convergence of numerical homogenization methods with windowing for general energy densities and general heterogeneities. In this setting, we are not able to exhibit error estimates, but we will prove convergence results and relate windowing methods to Tartar's correctors in homogenization.

3.3. A remark on boundary conditions. The mismatch between boundary conditions may be understood as follows. Let us consider a cubic domain $C(x, \eta)$ with $\eta / \epsilon \in \mathbb{N}$. The domain $C(x, \eta)$ is then exactly obtained by the concatenation of a given number of periodic cells. If periodic boundary conditions were used on $C(x, \eta), W_{\eta, \epsilon}$ would be exactly $W_{h o m}$ (since (2.3) would be exactly the cell problem). If Dirichlet boundary conditions are used, far from the boundary, the solution "tends to be" periodic. Close to the boundary the solution is very different from the periodic solution as illustrated in [2, Fig. 3]. In order to reduce the error, it is then natural to use the solution on periodic cells contained in $C(x, \eta)$ that are far from the boundary $\partial C(x, \eta)$. We refer the reader to the example of the half space dealt with in [3]. This remark is of great interest for the reconstruction of the fine scales features, which suffers highly from this boundary layer.

The technique of windowing is unlikely to change the rate of convergence for the homogenized coefficients, as can be easily seen in the periodic one-dimensional case (a direct calculation shows that the error still scales like $\frac{\epsilon}{\eta}$ and not better). However, not taking into account the boundary layer may improve the prefactor of the error. For the two-dimensional numerical examples considered in [21] and [10], namely a heterogeneous Laplace equation of type $-\operatorname{div} A_{\epsilon}(x) \nabla u=0$, with a $\epsilon$-periodic operator $A_{\epsilon}$ defined by

$$
A_{\epsilon}(x)=\left(\frac{2+1.8 \sin \left(2 \pi x_{1} / \epsilon\right)}{2+1.8 \cos \left(2 \pi x_{2} / \epsilon\right)}+\frac{2+\sin \left(2 \pi x_{2} / \epsilon\right)}{2+1.8 \cos \left(2 \pi x_{1} / \epsilon\right)}\right) \mathrm{Id},
$$

the associated energy density is $W_{\epsilon}(x, \xi)=\frac{1}{2} A_{\epsilon}(x) \xi \cdot \xi$. To be more precise Tables 3.1 and 3.2 report on some simple numerical tests that show the significant effect 
TABLE 3.1

Error on the approximated homogenized coefficients (performed with [14, FreeFEM] on a Laplace operator $\left(-\operatorname{div} A_{\epsilon} \nabla\right)$ with P2-finite elements on the cube $] 0,1\left[{ }^{2}\right.$, with 100 elements per periodic cell).

\begin{tabular}{|c|c|c|c|c|c|c|}
\hline $\begin{array}{c}\text { Number of periodic } \\
\text { cells per dimension }\end{array}$ & \multicolumn{3}{|c|}{ Without windowing } & \multicolumn{3}{c|}{ With windowing } \\
\hline & Error & $\begin{array}{c}\text { Rate of } \\
\text { convergence }\end{array}$ & $\begin{array}{c}\text { Prefactor } \\
\text { (rate=1) }\end{array}$ & Error & $\begin{array}{c}\text { Rate of } \\
\text { convergence }\end{array}$ & $\begin{array}{c}\text { Prefactor } \\
\text { (rate=1) }\end{array}$ \\
\hline 1 & 0.157 & - & 0.157 & 0.157 & - & 0.157 \\
\hline 2 & 0.0845 & 0.895 & 0.169 & 0.0210 & 2.90 & 0.0420 \\
\hline 4 & 0.0433 & 0.963 & 0.173 & 0.0118 & 0.835 & 0.0471 \\
\hline 8 & 0.0219 & 0.983 & 0.175 & 0.00597 & 0.979 & 0.0478 \\
\hline 12 & 0.0146 & 1.01 & 0.175 & 0.00397 & 1.00 & 0.0476 \\
\hline 16 & 0.0110 & 0.965 & 0.176 & 0.00299 & 0.985 & 0.0478 \\
\hline 20 & 0.00876 & 1.03 & 0.175 & 0.00239 & 1.00 & 0.0478 \\
\hline
\end{tabular}

TABLE 3.2

$L^{2}$-norm of the error on the corrector (performed with [14, FreeFEM] on a Laplace operator $\left(-\operatorname{div} A_{\epsilon} \nabla\right)$ with P2-finite elements on the cube $] 0,1{ }^{2}$, with 100 elements per periodic cell).

\begin{tabular}{|c|c|c|c|c|c|c|}
\hline $\begin{array}{c}\text { Number of } \\
\text { periodic cells } \\
\text { per dimension }\end{array}$ & \multicolumn{3}{|c|}{ Without windowing } & \multicolumn{3}{c|}{ With windowing } \\
\hline & Error & $\begin{array}{c}\text { Rate of } \\
\text { convergence }\end{array}$ & $\begin{array}{c}\text { Prefactor } \\
\text { (rate=0.5) }\end{array}$ & Error & $\begin{array}{c}\text { Rate of } \\
\text { convergence }\end{array}$ & $\begin{array}{c}\text { Prefactor } \\
\text { (rate=1) }\end{array}$ \\
\hline 1 & 0.210 & - & 0.210 & 0.210 & - & 0.210 \\
\hline 2 & 0.156 & 0.425 & 0.221 & 0.0116 & 0.893 & 0.0232 \\
\hline 4 & 0.113 & 0.468 & 0.226 & 0.00361 & 1.684 & 0.0144 \\
\hline 8 & 0.0808 & 0.484 & 0.229 & 0.00181 & 0.988 & 0.0145 \\
\hline 12 & 0.0662 & 0.491 & 0.229 & 0.00121 & 1.00 & 0.0145 \\
\hline 16 & 0.0574 & 0.496 & 0.230 & 0.000910 & 0.992 & 0.0146 \\
\hline 20 & 0.0515 & 0.492 & 0.230 & 0.000726 & 1.02 & 0.0145 \\
\hline
\end{tabular}

of windowing in the illustrative case (3.8). In Table 3.1 the error between the homogenized coefficients and the approximated homogenized coefficients is reported on, using Dirichlet boundary conditions on an increasing number of periodic cells. The first approximated operator is obtained without windowing, whereas the second one is obtained by considering only the periodic cell which is at the center of the domain of computation. The convergence rate is clearly proportional to the inverse of the number of periodic cells per dimension in both cases; however, the prefactor is four times smaller in the second case.

For the reconstruction of the fine scale features of the solution $u_{\epsilon}$, windowing reduces the $L^{2}$-norm of the error between the numerical corrector and the two-scale corrector (3.2), whose rate of convergence with respect to $\frac{\epsilon}{\eta}$ passes from $\frac{1}{2}$ to 1 using windowing, as illustrated in Table 3.2. This issue is dealt with theoretically in [21] and [8]. Its proof is based on the two-scale expansion of the solution and will not be detailed here since it cannot be generalized to other heterogeneities. In $[17$, sect. 3], the interest of windowing for nonlinear operators is addressed in terms of error contributions: the error made on the homogenized energy could be greater than the error due to the boundary layers of the corrector. Therefore the effect of windowing on the correctors may not change the order of the error for the fine scales. In this case, however, the prefactor of the error can still be reduced by windowing. 
3.4. A remark on the volume element $C(x, \eta)$. Assuming that the mismatch due to boundary conditions is reduced, one still has to deal with another source of error: the mismatch between $C(x, \eta)$ and the periodic cell. The domain $C(x, \eta)$ may not be exactly a multiple of the periodic cell. Therefore the mean of the energy on a periodic cell with a given periodic function does not coincide with the mean on $C(x, \eta)$ of the energy with the same periodic function. This error is more subtle than the previous one and of the same order, namely $\frac{\epsilon}{\eta}$ in (3.1) and for the the homogenized coefficients in the linear periodic case. This source of error is of a lower order for the Petrov-Galerkin formulation of the MsFEM [21], as will be discussed in the following subsection. This solution is intimately linked to the particular formulation (or discretization of $I_{\eta, \epsilon}$ in other terms) of the MsFEM. It does not apply to the HMM, for example. In the latter case, something else has to be done.

REMARK 3. In the case for which $C(x, \eta)$ is not exactly a multiple of the periodic cell, for both periodic and Dirichlet boundary conditions, numerical tests exhibit the same behavior as in Tables 3.1 and 3.2 .

3.5. Interpretation of the MsFEM in Petrov-Galerkin formulation. Let us first introduce some notation. Given a minimization problem

$$
\inf _{w \in V} \int_{\Omega} W(x, \nabla w)-f w
$$

its associated Euler-Lagrange equation

$$
-\operatorname{div} \partial_{\xi} W(x, \nabla w)=f,
$$

and two finite-dimensional spaces $V_{H}^{1}$ and $V_{H}^{2}$, we consider a discrete formulation: find $u_{H} \in V_{H}^{1}$ such that for all $w_{H} \in V_{H}^{2}$,

$$
\int_{\Omega} \partial_{\xi} W\left(x, \nabla u_{H}\right) \nabla w_{H}=\int_{\Omega} f w_{H} .
$$

We "abusively" say that the formulation is

- conforming if $V_{H}^{1} \subset V$ and nonconforming if $V_{H}^{1} \not \subset V$;

- Galerkin if $V_{H}^{1}=V_{H}^{2}$ and Petrov-Galerkin if $V_{H}^{1} \neq V_{H}^{2}$.

We also have to detail some kind of "generalized" variational formulation for the nonlinear MsFEM method. Let $V_{H}$ denote a classical $P 1$-FE space. For all $w_{H} \in V_{H}$, there exists a function $w_{H, \epsilon}^{M S F E M} \in L^{p}(\Omega)$ such that $\left.w_{H, \epsilon}^{M s F E M}\right|_{\mid Q_{H, i}} \in W^{1, p}\left(Q_{H, i}\right)$ for all $i$, and

$$
\int_{\Omega} W_{H, \epsilon}^{M s F E M}\left(x, \nabla w_{H}\right)=\int_{\Omega} W_{\epsilon}\left(x, \nabla w_{H, \epsilon}^{M s F E M}\right),
$$

where $\nabla w_{H, \epsilon}^{M S F E M}$ abusively denotes $\sum_{i} \nabla w_{H, \epsilon}^{M s F E M}{ }_{\mid Q_{H, i}} 1_{\mid Q_{H, i}}$. The correspondence (3.9) between $w_{H}$ and $w_{H, \epsilon}^{M s F E M}$ defines a (nonlinear) mapping from $V_{H}$ to $\oplus_{i} W^{1, p}\left(Q_{H, i}\right)$, as introduced in [11]. The nonlinear mapping provides us with a relationship of duality between the points of view $\left(W_{H, \epsilon}^{M s F E M}, V_{H}\right)$ and $\left(W_{\epsilon},\left\{w_{H, \epsilon}^{M s F E M}\right\}\right)$.

Without oversampling, the mapping takes values in $W^{1, p}(\Omega)$, whereas with oversampling the restrictions of $w_{H, \epsilon, \text { over }}^{M s F E M}$ belong to $W^{1, p}\left(Q_{H, i}\right)$ but $w_{H, \epsilon, \text { over }}^{M s F E M} \notin W^{1, p}(\Omega)$. The "generalized" variational formulation for the MsFEM then reads as follows: find $u_{H} \in V_{H}$ such that for all $w_{H} \in V_{H}$,

$$
\int_{\Omega} \partial_{\xi} W_{\epsilon}\left(x, \nabla u_{H, \epsilon}^{M s F E M}\right) \nabla w_{H, \epsilon}^{M s F E M}=\int_{\Omega} f w_{H, \epsilon}^{M s F E M},
$$

Copyright ( by SIAM. Unauthorized reproduction of this article is prohibited. 
where $u_{H, \epsilon}^{M S F E M}$ and $w_{H, \epsilon}^{M S F E M}$ are related to $u_{H}$ and $w_{H}$ by the nonlinear mapping (3.9). One can also define the following formulation: find $u_{H} \in V_{H}$ such that for all $w_{H} \in V_{H}$

$$
\int_{\Omega} \partial_{\xi} W_{\epsilon}\left(x, \nabla u_{H, \epsilon}^{M s F E M}\right) \nabla w_{H}=\int_{\Omega} f w_{H}
$$

According to the above definitions, formulation (3.10) is a Galerkin formulation, which is conforming for the classical MsFEM and nonconforming for the MsFEM with oversampling. Formulation (3.11) is a Petrov-Galerkin formulation, which is also conforming for the classical MsFEM and nonconforming for the MsFEM with oversampling.

In section 3.4, we have defined a "geometric error." The MsFEM does not suffer from this kind of error mainly because $\eta=H$ in the formulation. The proportion of each material at scale $\epsilon$ is globally conserved in $W_{H, \epsilon}^{M s F E M}$ due to (3.9). In other terms, if $W_{\epsilon}$ is the energy density of a periodic composition of two materials $A$ and $B$, the ratio of $A$ and $B$ in $W_{\epsilon}$ is exactly preserved in $W_{H, \epsilon}^{M s F E M}$, whereas it is preserved only up to an error of order $\frac{\epsilon}{\eta}$ for a generic averaged energy density $W_{\eta, \epsilon}$. Recalling the brief discussion in section 3.4, each periodic cell of the material $\Omega$ is exactly accounted for once in the MsFEM: if only half of a given periodic cell belongs to some $Q_{H, i}$, then the other half belongs to some other $Q_{H, j}$. For a generic $W_{\eta, \epsilon}$, this may not be true. In [21], the nonconforming Galerkin MsFEM of [10] is shown to still exhibit a cell resonance error for linear problems that can be reduced using a Petrov-Galerkin method. In the remainder of this section, we give a simple argument that may explain why the Petrov-Galerkin version of the nonconforming MsFEM is better in general than the Galerkin version. And we show how the nonconforming Petrov-Galerkin MsFEM may be recast within the analytic framework of section 2. In particular, without a Petrov-Galerkin formulation, there is no equality corresponding to (3.9) if oversampling is used (actually there is no variational interpretation; see Remark 5 hereafter).

To do so, let us study some basic properties of formulations (3.10) and (3.11) for both the classical and the "oversampled" MsFEM. We first consider the classical MsFEM. In this case, the following calculation,

$$
\begin{aligned}
\int_{\Omega} \partial_{\xi} W_{\epsilon}\left(x, \nabla u_{H, \epsilon}^{M s F E M}\right) \nabla w_{H, \epsilon}^{M s F E M} & =\sum_{i} \int_{Q_{H, i}} \partial_{\xi} W_{\epsilon}\left(x, \nabla u_{H, \epsilon}^{M s F E M}\right) \nabla w_{H, \epsilon}^{M s F E M} \\
& \left.=\sum_{i} \int_{Q_{H, i}} \partial_{\xi} W_{\epsilon}\left(x, \nabla u_{H, \epsilon}^{M s F E M}\right)\right)\left\langle\nabla w_{H}\right\rangle_{i} \\
& =\int_{\Omega} \partial_{\xi} W_{\epsilon}\left(x, \nabla u_{H, \epsilon}^{M s F E M}\right) \nabla w_{H}
\end{aligned}
$$

which holds due to the Euler-Lagrange equation associated with (2.12), shows that the differential terms of the variational formulations (3.10) and (3.11) indeed coincide.

On the contrary, for the "oversampled" MsFEM, the differential terms of the variational formulations (3.10) and (3.11) do not coincide. The Euler-Lagrange equation of (3.6) is no longer defined on $Q_{H, i}$ but on a larger domain $Q_{H+\zeta, i}$ which prevents us from writing the decomposition as a sum of Euler-Lagrange equations on $Q_{H, i}$, as is done in the previous calculation. Thus, for the "oversampled" method,

$$
\int_{\Omega} \partial_{\xi} W_{\epsilon}\left(x, \nabla u_{H, \epsilon, \text { over }}^{M s F E M}\right) \nabla w_{H, \epsilon, \text { over }}^{M s F E M} \neq \int_{\Omega} \partial_{\xi} W_{\epsilon}\left(x, \nabla u_{H, \epsilon, \text { over }}^{M s F E M}\right) \nabla w_{H}
$$

Copyright $($ ) by SIAM. Unauthorized reproduction of this article is prohibited. 
We actually have instead

$$
\begin{aligned}
\int_{\Omega} \partial_{\xi} W_{\epsilon}\left(x, \nabla u_{H, \epsilon, \text { over }}^{M s F E M}\right) \nabla w_{H, \epsilon, \text { over }}^{M s F E M} \\
=\sum_{i} \int_{Q_{H, i}} \partial_{\xi} W_{\epsilon}\left(x, \nabla u_{H, \epsilon, \text { over }}^{M s F E M}\right) \nabla w_{H, \epsilon, \text { over }}^{M s F E M} \\
=\sum_{i} \int_{Q_{H, i}} \partial_{\xi} W_{\epsilon}\left(x, \nabla u_{H, \epsilon, \text { over }}^{M s F E M}\right)\left(\left\langle\nabla w_{H}\right\rangle_{i}+\nabla w_{\epsilon, \text { over }}^{H, i}\right) \\
=\sum_{i} \int_{Q_{H, i}} \partial_{\xi} W_{\epsilon}\left(x, \nabla u_{H, \epsilon, \text { over }}^{M s F E M}\right)\left\langle\nabla w_{H}\right\rangle_{i} \\
\quad-\sum_{i} \int_{Q_{H+\zeta, i} \backslash Q_{H, i}} \partial_{\xi} W_{\epsilon}\left(x, \nabla u_{H, \epsilon, \text { over }}^{M s F M}\right) \nabla w_{\epsilon, \text { over }}^{H, i},
\end{aligned}
$$

formally using the Euler-Lagrange equation associated with (3.6):

$$
\int_{Q_{H+\zeta, i}} \partial_{\xi} W_{\epsilon}\left(y, \nabla u_{H, \epsilon, \text { over }}^{M s F E M}\right) \cdot \nabla w_{\epsilon, \text { over }}^{H, i}=0
$$

where $\nabla w_{\epsilon, \text { over }}^{H, i}$ is given by (3.7). The difference in the differential operator between the Petrov-Galerkin and the Galerkin formulations of the "oversampled" MsFEM (which are both nonconforming) is therefore given by

$$
\sum_{i} \int_{Q_{H+\zeta, i} \backslash Q_{H, i}} \partial_{\xi} W_{\epsilon}\left(x, \nabla u_{H, \epsilon}^{M s F E M}\right) \nabla w_{\epsilon, \text { over }}^{H, i} .
$$

It is now time to come back to the origin of windowing methods. Windowing aims at getting rid of the effects of the boundary layer on $Q_{H, i}$ by computing the minimum (3.4) on a domain $Q_{H+\zeta, i}$ of diameter of order $H+\zeta$ and by considering only the restriction of the associated solution $w_{\epsilon, \text { over }}^{H, i} \in W^{1, p}\left(Q_{H+\zeta, i}\right)$ on $Q_{H, i}$. Our simple calculation shows that the nonconforming Galerkin MsFEM implicitly takes into account the term (3.13). This term involves the restriction of the multiscale finite element on $Q_{H+\zeta, i} \backslash Q_{H, i}$, which contains a part of the boundary layer that was supposed to be cancelled by the windowing method. Erasing the very last term of (3.12) and going backwards, we recover the nonconforming Petrov-Galerkin formulation of the MsFEM, which truly avoids the boundary layer.

To conclude this section, let us show that the nonconforming Petrov-Galerkin formulation of the MsFEM is equivalent to the variational formulation recalled in section 2 combined with the windowing introduced in section 3.2. Using the results of $[18$, sect. 4] to switch the derivation with respect to $\xi$ and the minimization (3.4) for convex energies with invertible Hessians (and proceeding formally otherwise), one may write the nonconforming Petrov-Galerkin MsFEM as follows:

$$
\begin{aligned}
\int_{\Omega} \partial_{\xi} W_{\epsilon}\left(x, \nabla u_{H, \epsilon, \text { over }}^{M S F E M}\right) \nabla w_{H} & =\sum_{i} \int_{Q_{H, i}} \partial_{\xi} W_{\epsilon}\left(x, \nabla u_{H, \epsilon, \text { over }}^{M s F E M}\right)\left\langle\nabla w_{H}\right\rangle_{i} \\
& =\sum_{i} \int_{Q_{H, i}} \partial_{\xi} W_{H, \epsilon, \text { over }}^{M s F E M}\left(x,\left\langle\nabla u_{H}\right\rangle_{i}\right)\left\langle\nabla w_{H}\right\rangle_{i} \\
& =\int_{\Omega} \partial_{\xi} W_{H, \epsilon, \text { over }}^{M s F E M}\left(x, \nabla u_{H}\right) \nabla w_{H}
\end{aligned}
$$

Copyright $@$ by SIAM. Unauthorized reproduction of this article is prohibited. 
In section 4, we will prove the convergence of the Petrov-Galerkin formulation of the "oversampled" MsFEM for rather general elliptic operators and general heterogeneities using the variational formulation of section 2 .

REMARK 4. Let us stress the fact that the previous calculation shows that the nonconforming Petrov-Galerkin formulation of the MsFEM (which yields discontinuous tests functions) for $W_{\epsilon}$ can be equivalently seen as a Galerkin method for $W_{H, \epsilon, o v e r}^{M S F E M}$. This explains why variational methods can be used on $W_{H, \epsilon, o v e r}^{M s F E M}$ to prove the convergence of the PG-MsFEM for $W_{\epsilon}$.

REMARK 5. The nonconforming Galerkin formulation of the MsFEM cannot be recast within the framework of section 2 .

4. Windowing for general heterogeneities. In this section, we first define the windowing method for general heterogeneities by making precise the dependence of the windowing upon the parameter $\epsilon$ and the characteristic lengthscale $\eta$. Provided a right scaling, we then prove the convergence of numerical homogenization methods with windowing, within the framework of section 2 .

There is numerical evidence that shows the practical interest of windowing for nonperiodic problems. There is also another motivation that is related to Tartar's corrector. In section 2.4, the numerical corrector has been related to Tartar's corrector, provided an approximation depending on $H$ and provided $\omega=\omega_{1}$. In Tartar's original work, however, the correctors are proved to exist using $\omega \subset \subset \omega_{1}$, which is windowing in the present language. Numerical correctors with windowing are therefore approximations of Tartar's correctors that may seem more natural than the numerical correctors of section 2.4. The use of windowing allows us to recover all the diversity of the original Tartar correctors.

4.1. Scaling of the windowing. The aim of windowing is to reduce the mismatch between the free oscillations of an unconstrained solution at fixed $\epsilon$ and the boundary conditions on domains $C(x, \eta)$. A major assumption concerns the convergence of the energies $I_{\epsilon}$ to a homogenized energy $I_{h o m}$ which is not supposed to exhibit oscillations at small scales. The windowing for general heterogeneities should match the scales of the oscillations. Therefore it has to vanish with $\eta$, but it may also already vanish with $\epsilon$. We set the following.

Definition 4. Let $\zeta: \mathbb{R}_{+} \rightarrow \mathbb{R}_{+}$. For all $x \in \Omega, \eta>0$, and $\epsilon<\eta$, $\zeta$ defines an $\epsilon$-admissible windowing domain $C(x, \eta+\zeta(\epsilon))$ if

$$
\lim _{\epsilon \rightarrow 0} \zeta(\epsilon)=0
$$

and an $\eta$-admissible windowing domain $C(x, \eta+\zeta(\eta))$ if

$$
\lim _{\eta \rightarrow 0} \frac{\zeta(\eta)}{\eta}=0
$$

Heuristically, if $\epsilon$ measures the typical "size" of the heterogeneities, $\zeta$ should satisfy $\lim _{\epsilon \rightarrow 0} \zeta(\epsilon)=0$ and a property of the type $\lim _{\epsilon \rightarrow 0} \frac{\zeta(\epsilon)}{\epsilon}=+\infty$ in order to see the effect of windowing. The prototypical example is given by the linear periodic case in section 3.3 for which the boundary layer is of order $\epsilon$ (thus any $\zeta(\epsilon)=\epsilon^{\alpha}$ with $1>\alpha>0$ is enough).

The above heuristics is related to geometric properties of the heterogeneities (in particular the period, or the correlation length). Hence, $\epsilon$ should ideally be a typical 
lengthscale, whereas it appears as a simple parameter (which has no geometric meaning) for the assumption $I_{\epsilon} \Gamma\left(L^{p}\right)$-converges to $I_{h o m}$. In Definition 4, we thus consider only windowings that are "stable under" a change of parametrization.

In the following subsections, we prove that the use of admissible windowings does not affect the convergence of the numerical homogenization method, meaning that the method also converges using windowing.

REMARK 6. The windowings introduced in Definition 4 are two extreme cases. One can also introduce particular windowings depending both on $\epsilon$ and $\eta$ and providing us with suitable regimes for given applications. They can also be seen as particular cases of the $\eta$-admissible windowing.

4.2. Convergence results. In this section, we prove the convergence of numerical homogenization with $\eta$-admissible windowing, which also implies the convergence with $\epsilon$-admissible windowing. We first address the convergence of a continuous "windowed" energy density whose FE discretization leads to the HMM. We then show the convergence of two versions of the nonconforming Petrov-Galerkin MsFEM.

4.2.1. Convergence at the continuous level. The "windowed" continuous energy density is given by the following.

Definition 5. Let $\zeta$ be an $\eta$-admissible windowing and $W_{\epsilon}$ satisfy $\mathrm{H} 1, \mathrm{H} 4$, and H3. For all $\eta, \epsilon>0$, the associated "windowed" energy density is defined by

$$
\mathcal{W}_{\eta, \epsilon}^{\text {win }}(x, \xi)=\left\langle W_{\epsilon}\left(y, \xi+\nabla v_{\eta, \epsilon}^{\text {win }}\right)\right\rangle_{C(x, \eta)},
$$

where $v_{\eta, \epsilon}^{\text {win }}$ is the restriction on $C(x, \eta)$ of $\tilde{v}_{\eta, \epsilon}^{\text {win }}$, the solution of (3.4) with $\zeta=\zeta(\eta)$.

We then have the following two convergence results.

THEOREM 4. Let $W_{\epsilon}$ satisfy $\mathrm{H} 1, \mathrm{H} 2$ (strictly), and $\mathrm{H} 3$ uniformly for $p>1$ and $\zeta$ be an $\eta$-admissible windowing; then the energy densities $C \mathcal{W}_{\eta, \epsilon}^{\text {win }}$ also satisfy $\mathrm{H} 1, \mathrm{H} 2$, and $\mathrm{H} 3$ for $\eta$ small enough, and the energy $I_{\eta, \epsilon}^{\text {win }}: v \in W^{1, p}(\Omega) \mapsto$ $\int_{\Omega} C \mathcal{W}_{\eta, \epsilon}^{\text {win }}(x, \nabla v) \Gamma\left(L^{p}\right)$ - and $\Gamma\left(W^{1, p}\right)$-converges to $I_{\text {hom }}$ as $\epsilon$ and $\eta$ go to 0 , where $C \mathcal{W}$ denotes the convex envelope of $\mathcal{W}$. Therefore, any sequence $u_{\eta, \epsilon}^{\text {win }}$ of minimizers of $\inf \left\{I_{\eta, \epsilon}^{\text {win }}(v) \mid v \in W^{1, p}(\Omega, \mathbb{R})+B C\right\}$ strongly converges to the unique minimizer $u_{\text {hom }}$ of $\inf \left\{I_{\text {hom }}(v) \mid v \in W^{1, p}(\Omega, \mathbb{R})+B C\right\}$ in $W^{1, p}\left(\Omega, \mathbb{R}^{n}\right)$.

TheOrem 5. Let $W_{\epsilon}$ satisfy $\mathrm{H} 1, \mathrm{H} 4$, and $\mathrm{H} 3$ uniformly for $p>1$ and $\zeta$ be an $\eta$-admissible windowing; then the energy densities $Q \mathcal{W}_{\eta, \epsilon}^{\text {win }}$ are standard energy densities, and $I_{\eta, \epsilon}^{\text {win }}: v \in W^{1, p}(\Omega) \mapsto \int_{\Omega} Q \mathcal{W}_{\eta, \epsilon}^{\text {win }}(x, \nabla v) \Gamma\left(L^{p}\right)$ - and $\Gamma\left(W^{1, p}\right)$-converges to $I_{\text {hom }}$ as $\epsilon$ and $\eta$ go to 0 . Therefore, for any sequence $u_{\eta, \epsilon}^{\text {win }}$ of minimizers of $\inf \left\{I_{\eta, \epsilon}^{\text {win }}(v) \mid v \in W^{1, p}\left(\Omega, \mathbb{R}^{d}\right)+B C\right\}$, there exists a minimizer $u_{\text {hom }}$ of $\inf \left\{I_{\text {hom }}(v) \mid\right.$ $\left.v \in W^{1, p}\left(\Omega, \mathbb{R}^{d}\right)+B C\right\}$ such that

$$
\lim _{\eta \rightarrow 0} \lim _{\epsilon \rightarrow 0} u_{\eta, \epsilon}^{\text {win }}=u_{\text {hom }} \quad \text { weakly in } W^{1, p}\left(\Omega, \mathbb{R}^{d}\right)
$$

up to extraction.

In practice, one does not need to convexify $\mathcal{W}_{\eta, \epsilon}^{\text {win }}$ since the minimum is searched in a finite-dimensional subspace of $W^{1, p}(\Omega)$, the strict convexity being recovered at the limit $\epsilon \rightarrow 0$ for $\epsilon$-windowings and $\eta \rightarrow 0$ for $\eta$-windowings, in the spirit of $[17$, Thm. 4] for the quasiconvex case.

Theorems 4 and 5 imply the convergence of the HMM with windowing in the general case.

Proof of Theorems 4 and 5. We divide the proof into two steps. We first introduce an averaged energy density for which the strategy used to prove [17, Theorems 1 and 3] 
holds. We then show the "windowed" energy density to be uniformly close to this averaged energy as $\eta$ goes to zero.

Let us introduce the averaged energy density

$$
\tilde{\mathcal{W}}_{\eta, \epsilon}^{w i n}(x, \xi)=\left\langle W_{\epsilon}\left(y, \xi+\nabla \tilde{v}_{\eta, \epsilon}^{w i n}\right)\right\rangle_{C(x, \eta+\zeta(\eta))}
$$

This energy density is of type (2.3) (cf. Remark 1) up to denoting by $\tilde{\eta}=\eta+\zeta(\eta)$. Thus Theorems 1 and 2 apply, and we denote by $\tilde{I}_{\eta, \epsilon}^{\text {win }}$ the associated energy functional.

Let us now prove that the $\Gamma\left(L^{p}\right)$ - and $\Gamma\left(W^{1, p}\right)$-convergence of $\tilde{I}_{\eta, \epsilon}^{\text {win }}$ and $I_{\eta, \epsilon}^{\text {win }}$ are equivalent. Due to Meyers' regularity estimate, H1, H4, H3, and possibly a convolution argument (see [22] and [5, Thm. C.2]), there exist $\alpha>0$ and $c>0$, independent of $\eta$ and $\epsilon$, such that

$$
\left\|\tilde{v}_{\eta, \epsilon}^{w i n}\right\|_{W^{1, p+\alpha}(C(x, \eta+\zeta(\eta)))} \leq c\left\|\tilde{v}_{\eta, \epsilon}^{w i n}\right\|_{W^{1, p}(C(x, \eta+\zeta(\eta)))} .
$$

The coefficients (exponent $\alpha$ and prefactor $c$ ) appearing in the Meyers' estimate depend only on the growth conditions and on the domain (see [16] and [15, Thm. 3.1 and Rem. 3.5]). Let us prove that they do not depend on $\eta$ either. Up to introducing the scaling

$$
W_{\#}^{1, p}\left((0,1)^{n}\right) \ni v \mapsto \bar{v}(\cdot)=(\eta+\zeta(\eta)) v\left(\frac{\cdot}{\eta+\zeta(\eta)}\right) \in W_{\#}^{1, p}\left((0, \eta+\zeta(\eta))^{n}\right),
$$

we have

$$
\int_{(0,1)^{n}} W\left(x, \nabla_{x} v(x)\right) d x=\frac{1}{(\eta+\zeta(\eta))^{n}} \int_{(0, \eta+\zeta(\eta))^{n}} W\left(\frac{y}{\eta+\zeta(\eta)}, \nabla_{y} \bar{v}(y)\right) d y
$$

for any standard energy density. Let $c_{1}$ denote the coefficient provided by Meyers' theorem on the domain $(0,1)^{n}$ and by $c_{2}$ the constant of the Poincaré-Wirtinger inequality. Let $v \in W_{\#}^{1, p}(0,1)^{n}$ be a minimizer of the associated energy on a given set. We have for $\eta+\zeta(\eta) \leq 1$

$$
\begin{aligned}
\|\bar{v}\|_{W^{1, p+\alpha}\left((0, \eta+\zeta(\eta))^{n}\right)}^{p+\alpha} & =(\eta+\zeta(\eta))^{n+p+\alpha}\|v\|_{L^{p+\alpha}\left((0,1)^{n}\right)}^{p+\alpha}+(\eta+\zeta(\eta))^{n}\|\nabla v\|_{L^{p+\alpha}\left((0,1)^{n}\right)}^{p+\alpha} \\
& \leq(\eta+\zeta(\eta))^{n}\|v\|_{W^{1, p+\alpha}\left((0,1)^{n}\right)}^{p+\alpha} \\
& \leq(\eta+\zeta(\eta))^{n} c_{1}^{p+\alpha}\|v\|_{W^{1, p}\left((0,1)^{n}\right)}^{p+\alpha} \\
& \leq(\eta+\zeta(\eta))^{n} c_{1}^{p+\alpha}\left(1+c_{2}\right)^{p+\alpha}\|\nabla v\|_{L^{p}\left((0,1)^{n}\right)}^{p+\alpha} \\
& \leq c_{1}^{p+\alpha}\left(1+c_{2}\right)^{p+\alpha}\|\bar{v}\|_{W^{1, p}\left((0, \eta+\zeta(\eta))^{n}\right)}^{p+\alpha},
\end{aligned}
$$

which shows that (4.2) holds with $c=c_{1}\left(1+c_{2}\right)$.

Using the growth condition $\mathrm{H} 3$ on $W_{\epsilon},(3.4)$, and (4.2) we obtain

$$
\left\|\tilde{v}_{\eta, \epsilon}^{w i n}\right\|_{W^{1, p+\alpha}(C(x, \eta+\zeta(\eta)))}^{p} \leq c(\eta+\zeta(\eta))^{n}\left(1+|\xi|^{p}\right) .
$$

The application of the Hölder inequality yields

$$
\left\|\tilde{v}_{\eta, \epsilon}^{w i n}\right\|_{W^{1, p}(C(x, \eta+\zeta(\eta)) \backslash C(x, \eta))}^{p} \leq C\left[\eta^{n-1} \zeta(\eta)\right]^{\frac{\alpha}{p+\alpha}}\left(\left\|\tilde{v}_{\eta, \epsilon}^{w i n}\right\|_{W^{1, p+\alpha}(C(x, \eta+\zeta(\eta)))}^{p}\right),
$$

which implies that

$$
\left\|\tilde{v}_{\eta, \epsilon}^{w i n}\right\|_{\left.W^{1, p}(C(x, \eta+\zeta(\eta)) \backslash C(x, \eta))\right)}^{p} \leq C(\eta+\zeta(\eta))^{n \frac{p}{p+\alpha}}\left[\eta^{n-1} \zeta(\eta)\right]^{\frac{\alpha}{p+\alpha}}\left(1+|\xi|^{p}\right)
$$

Copyright (C) by SIAM. Unauthorized reproduction of this article is prohibited. 
using (4.3) and noticing that $(\eta+\zeta(\eta))^{n \frac{p}{p+\alpha}} \geq(\eta+\zeta(\eta))^{n}$. We finally deduce that

$$
\left|\tilde{\mathcal{W}}_{\eta, \epsilon}^{\text {win }}(x, \xi)-\mathcal{W}_{\eta, \epsilon}^{\text {win }}(x, \xi)\right| \leq C\left(\left[\frac{\zeta(\eta)}{\eta}\right]^{\frac{\alpha}{p+\alpha}}+\frac{\zeta(\eta)}{\eta}\right)\left(1+|\xi|^{p}\right)
$$

using the well-known quasi-uniform Lipschitz property of rank-one convex functions (see $\left[17\right.$, eq. (2.12)], e.g.) and noticing that $\frac{\eta}{\eta+\zeta}=\eta\left(1-\frac{\zeta}{\eta}+o\left(\frac{\zeta}{\eta}\right)\right)$ and

$$
\frac{(\eta+\zeta(\eta))^{n \frac{p}{p+\alpha}}}{(\eta+\zeta(\eta))^{n}}\left[\eta^{n-1} \zeta(\eta)\right]^{\frac{\alpha}{p+\alpha}}=(\eta+\zeta(\eta))^{-n \frac{\alpha}{p+\alpha}}\left[\eta^{n-1} \zeta(\eta)\right]^{\frac{\alpha}{p+\alpha}} \leq\left[\frac{\zeta(\eta)}{\eta}\right]^{\frac{\alpha}{p+\alpha}} .
$$

In particular, (4.4) implies that $\mathcal{W}_{\eta, \epsilon}^{\text {win }}$ satisfies $\mathrm{H} 3$ with a modified but strictly positive constant $c$ for $\eta$ small enough (since $\tilde{\mathcal{W}}_{\eta, \epsilon}^{\text {win }}$ does), which ensures the existence of minimizers for the relaxed problem.

The dominated convergence theorem then allows us to prove the uniform convergence to zero of $\tilde{I}_{\eta, \epsilon}^{\text {win }}-I_{\eta, \epsilon}^{\text {win }}$ on any bounded subset of $W^{1, p}(\Omega)$ as $\eta$ goes to zero. This is enough to ensure the equivalence of the $\Gamma$-convergences of the energy functionals, as briefly recalled below (see [4] or [6] for classical definitions related to $\Gamma$-convergence).

Let us first notice that the energies are finite only on $W^{1, p}(\Omega)$. For all $w \in$ $W^{1, p}(\Omega)$ and all sequences $w_{\eta, \epsilon} \in W^{1, p}(\Omega)$ such that $\lim _{\eta \rightarrow 0} \lim _{\epsilon \rightarrow 0} w_{\eta, \epsilon}=w$ in $L^{p}(\Omega)$, either

$$
\lim _{\eta \rightarrow 0} \lim _{\epsilon \rightarrow 0} \tilde{I}_{\eta, \epsilon}^{\text {win }}\left(w_{\eta, \epsilon}\right)=\lim _{\eta \rightarrow 0} \lim _{\epsilon \rightarrow 0} I_{\eta, \epsilon}^{\text {win }}\left(w_{\eta, \epsilon}\right)=+\infty
$$

and the $\Gamma$-liminf inequality trivially holds, or the sequence $\left\{w_{\epsilon, \eta}\right\}$ is bounded in $W^{1, p}(\Omega)$. In the latter case, the sequence belongs to a set on which the convergence of $\tilde{I}_{\eta, \epsilon}^{\text {win }}-I_{\eta, \epsilon}^{\text {win }}$ to zero is uniform. Thus, $\lim _{\eta \rightarrow 0} \lim _{\epsilon \rightarrow 0} \tilde{I}_{\eta, \epsilon}^{\text {win }}\left(w_{\eta, \epsilon}\right)-I_{\eta, \epsilon}^{\text {win }}\left(w_{\eta, \epsilon}\right)=0$ and the $\Gamma\left(L^{p}\right)$-liminf (resp., limsup) of $I_{\eta, \epsilon}^{w i n}$ and $\tilde{I}_{\eta, \epsilon}^{\text {win }}$ coincide. Therefore they have the same $\Gamma\left(L^{p}\right)$-limit. The same reasoning holds for the $\Gamma\left(W^{1, p}\right)$-convergence.

As a consequence, the $\Gamma$-convergence results obtained for $\tilde{I}_{\eta, \epsilon}^{\text {win }}$ hold for $I_{\eta, \epsilon}^{w i n}$, which concludes the proof of Theorems 4 and 5 .

\subsubsection{Convergence of the nonconforming Petrov-Galerkin MsFEM.}

DeFINITION 6. Let $\zeta$ be an $\eta$-admissible windowing and $W_{\epsilon}$ satisfy $\mathrm{H} 1, \mathrm{H} 4$, and H3. Let $\left\{Q_{H, i}\right\}_{i}$ be a triangulation of $\Omega$. For all $\eta, \epsilon>0$, the associated MsFEM "oversampled" energy density is defined by

$$
W_{H, \epsilon, \text { over }}^{M s F E M}(x, \xi)=\sum_{i}\left\langle W_{\epsilon}\left(y, \xi+\nabla v_{\epsilon, \text { over }}^{H, i}\right)\right\rangle_{Q_{H, i}} 1_{Q_{H, i}}(x),
$$

where $v_{\epsilon, \text { over }}^{H, i}$ is a solution of (3.6).

The associated result is then the following.

TheOrem 6. Let $W_{\epsilon}$ satisfy $\mathrm{H} 1, \mathrm{H} 4$ (resp., $\mathrm{H} 2$ strictly), and $\mathrm{H} 3$ uniformly for $p>1$ and $\zeta$ be an $\eta$-admissible windowing. Let $V_{H}$ be the space of P1-finite elements on the regular triangulation $\left\{Q_{H, i}\right\}_{i}$. The "oversampled" Petrov-Galerkin MsFEM reads

$$
\begin{aligned}
\inf \left\{\sum_{i=1}^{I_{H}}\left|Q_{H, i}\right| W_{H, \epsilon, \text { over }}^{M s F E M}\left(x_{i}, \nabla u_{H}\left(x_{i}\right)\right)\right. & \\
& \left.-\sum_{i=1}^{I_{H}} \sum_{j=1}^{N_{G P}} q_{j} f\left(x_{i j}\right) u_{H}\left(x_{i j}\right), u_{H} \in V_{H}+B C\right\},
\end{aligned}
$$

Copyright $@$ by SIAM. Unauthorized reproduction of this article is prohibited. 
where the second term $f \in L^{p^{\prime}}(\Omega)$ of the energy has been integrated by a quadrature rule associated with the triangulation. Then any sequence of solutions $\left\{u_{\epsilon, H}^{\text {over }}\right\}$ to (4.5) converges weakly in $W^{1, p}(\Omega)$ up to extraction (resp., converges strongly in $W^{1, p}(\Omega)$ ) to a minimizer (resp., the unique minimizer) of $w \mapsto I_{h o m}(w)-\int_{\Omega} f w$ on $W^{1, p}(\Omega)+B C$.

Proof of Theorem 6. Let us divide the proof into three steps. First, we introduce an averaged energy density whose associated energy functional $\Gamma\left(W^{1, p}\right)$-converges to the homogenized energy. We then prove the convergence of the associated infima to the infimum of the homogenized energy, following the proof of [17, sect. 3.2]. This implies the results of Theorem 6 for this averaged energy. Finally, we apply the argument of uniform convergence used in the proof of Theorems 4 and 5 .

Let us consider the following averaged energy density:

$$
\tilde{W}_{H, \epsilon, \text { over }}^{M s F E M}(x, \xi)=\sum_{i}\left\langle W_{\epsilon}\left(y, \xi+\nabla v_{\epsilon, \text { over }}^{H, i}\right)\right\rangle_{Q_{H+\zeta(H), i}} 1_{Q_{H, i}}(x) .
$$

Let $P_{H}$ be an equicontinuous family of projectors from $W^{1, p}(\Omega)$ to $V_{H}$ such that for all $w \in W^{1, p}(\Omega), \lim _{H \rightarrow 0}\left\|P_{H} w-w\right\|_{W^{1, p}(\Omega)}=0$. We then associate with $\tilde{W}_{H, \epsilon, \text { over }}^{M s F E M}$ an energy functional $\tilde{I}_{H, \epsilon, \text { over }}^{M s F E M}: W^{1, p}(\Omega) \rightarrow \mathbb{R}$ defined by

$$
\tilde{I}_{H, \epsilon, \text { over }}^{M s F E M}(w)=\int_{\Omega} \tilde{W}_{H, \epsilon, \text { over }}^{M s F E M}\left(x, \nabla P_{H} w\right) .
$$

This family of energy functionals is equicontinuous on $W^{1, p}(\Omega)$ (see [17, p. 1033]) and converges pointwise on $W^{1, p}(\Omega)$ to $I_{\text {hom }}$ as $\epsilon$ and $H$ vanish. Thus [17, Lem. 8] (or [6, Thm. 5.9]) implies the $\Gamma\left(W^{1, p}\right)$-convergence of $\tilde{I}_{H, \epsilon, \text { over }}^{M s F E M}$ to $I_{\text {hom }}$. It remains to prove the convergence of the infima of $\tilde{I}_{H, \epsilon, \text { over }}^{M s F E M}$ to the infimum of the homogenized energy to obtain the thesis of Theorem 6 for the family $\tilde{I}_{H, \epsilon, o v e r}^{M S F E M}$. We treat in detail only the new argument (based on Meyers' estimates) with respect to [17, pp. 10331035]. It is enough to prove that $\tilde{I}_{H, \epsilon, o v e r}^{M S F E M}-I_{H, \epsilon}^{M s F E M}$ converges uniformly to zero on bounded subsets of $W^{1, p}(\Omega)$. To this aim, we can apply Meyers' estimates on each mesh element $Q_{H, i}$. The exponent $\alpha$ in (4.2) may, however, depend on $H$. Due to the regularity of the mesh this is not the case, and there exists $\bar{\alpha}$ independent of $H$ such that Meyers' estimate holds on every $Q_{H, i}$ with exponent $\bar{\alpha}$.

It suffices to introduce a linear transformation $T_{H, i}$ which maps the reference mesh element $Q$ onto $Q_{H, i}$. Then let us denote by $\left(\lambda_{k}\right)$ the eigenvalues of $T_{H, i}$. Up to a change of variable using $T_{H, i}^{-1}$ and an isotropic dilatation by a factor $\sqrt[n]{\operatorname{det} T_{H, i}^{-1}}$,

$$
W_{0}^{1, p}\left(Q_{H, i}\right) \ni \bar{v} \mapsto v(\cdot)=\left(\sqrt[n]{\operatorname{det} T_{H, i}^{-1}}\right) \bar{v}\left(T_{H, i} \cdot\right) \in W_{0}^{1, p}(Q),
$$

we have

$$
\int_{Q_{H, i}} W\left(y, \nabla_{y} \bar{v}(y)\right) d y=\operatorname{det} T_{H, i} \int_{Q} W\left(T_{H, i} x, \frac{T_{H, i}^{-1}}{\sqrt[n]{\operatorname{det} T_{H, i}^{-1}}} \nabla_{x} v(x)\right) d x
$$

for any standard energy density. The "effective" energy density

$$
W^{H, i}(x, \xi)=W\left(T_{H, i} x, \frac{T_{H, i}^{-1}}{\sqrt[n]{\operatorname{det} T_{H, i}^{-1}}} \xi\right)
$$

Copyright ( by SIAM. Unauthorized reproduction of this article is prohibited. 
on $Q$ satisfies a growth condition of order $p$ with constants depending only on $c, C$, and the quotients $\left\{\frac{\lambda_{k}}{\sqrt[n]{\operatorname{det} T_{H, i}}}\right\}_{k \in \llbracket 1, n \rrbracket}$. These quotients are bounded from above and below uniformly in $H$ and $i$ by definition of the regularity of the mesh. Therefore, there exists $\bar{\alpha}$ associated with the reference mesh element and this growth condition, such that Meyers' estimate holds on all $Q_{H, i}$. The strategy used in the proof of Theorem 4 then shows the uniform convergence of $\tilde{I}_{H, \epsilon, \text { over }}^{M s s E M}-I_{H, \epsilon}^{\text {over }}$ to zero on bounded subsets of $W^{1, p}(\Omega)$, which implies the convergence of the infimum of $\tilde{I}_{H, \epsilon, \text { over }}^{M s F E M}$ to the limit of the infima of $I_{H, \epsilon}^{M S F E M}$, which is exactly the infimum of $I_{h o m}$ as proved in [17, pp. 1033-1035]. The results of Theorem 6 then hold for the energy density $\tilde{W}_{H, \epsilon \text {,over }}^{M S F}$.

As for the proof of Theorem 4 we use Meyers' estimate once more to obtain the uniform convergence of $\tilde{I}_{H, \epsilon, \text { over }}^{M s F E M}-I_{H, \epsilon, \text { over }}^{M s F E M}$ to zero on bounded subsets of $W^{1, p}(\Omega)$, proving Theorem 6 .

REMARK 7. In [21], the "oversampled" energy density is defined in a slightly different way. In the same spirit, one can replace $v_{H, \epsilon}^{\text {over }, i}$ by $\bar{v}_{H, \epsilon}^{\text {over, } i}(y)=v_{H, \epsilon}^{\text {over }, i}(y)-$ $\left\langle\nabla v_{H, \epsilon}^{\text {over,i }}\right\rangle_{Q_{H, i}} \cdot y$. In particular, this formulation satisfies Hill's lemma, which is widely used in mechanics. The present proof easily adapts since

$$
\lim _{H \rightarrow 0} \lim _{\epsilon \rightarrow 0}\left\langle\nabla v_{H, \epsilon}^{o v e r, i}\right\rangle_{Q_{H, i}}=0
$$

and $W_{\epsilon}(y, \cdot)$ is uniformly (in space) Lipschitz-continuous.

4.3. Fine scale reconstruction. We now extend the numerical corrector of $[17$, Def. 5] to the case of windowing. In the linear periodic case, windowing improves the approximation a lot since a great part of the error is located in a boundary layer of order $\epsilon$. For general heterogeneities we are not able to show that the approximation is better. Even if it were, in view of [17, sect. 3.2], it is not clear whether the order of the global error is reduced. It also seems delicate to generalize the estimates derived in [10], [21] to monotone operators in a periodic setting since the analysis of the correctors is far less complete in this case than for linear problems. In particular, the starting point of [10], [21], for the periodic linear case, is the multiple scale expansion $[10$, eq. $(2.7)]$

$$
u_{\epsilon}=u_{0}+\epsilon \chi^{i}\left(\frac{x}{\epsilon}\right) \nabla_{i} u_{0}+\epsilon \theta^{u_{0}},
$$

where $u_{0}$ is the solution of the homogenized problem, $\left(\chi^{i}\right)_{i}$ is the corrector vector, and $\theta^{u_{0}}$ accounts for the mismatch of boundary conditions. Such an expansion requires $\chi^{i}(\dot{\bar{\epsilon}}) \nabla_{i} u_{0} \in W^{1, p}(\Omega)$, which does not hold in general due to optimal regularity results by Savaré in [27]. Actually, $u_{0} \in W^{1+2 / p-\eta, p}$ for all $\eta>0$ and not $u_{0} \in W^{2, \infty}$ in general.

We prove, however, that the numerical corrector associated with the windowing method has the same general convergence properties as the numerical corrector without windowing. The interest of windowing then relies on the possible reduction of the prefactor term in the error. Its efficiency is illustrated numerically in [12, p. 67] for a linear stochastic case.

Definition 7. Let $\left\{Q_{H, i}\right\}_{i \in \llbracket 1, I_{H} \rrbracket}$ be as in Definition 3. Keeping the notation of Theorem 4 , we define the numerical correctors $v_{\eta, \epsilon, \text { win }}^{H, i}$ for a strictly convex energy density as the restriction on $Q_{H, i}$ of the unique minimizers (up to a constant) of 
$\inf \left\{\int_{Q_{H+\zeta(\eta), i}} W_{\epsilon}(x, \nabla v) \mid v \in W^{1, p}\left(Q_{H+\zeta(H), i}\right), v(y)=\left\langle\nabla u_{\eta, \epsilon}^{w i n}\right\rangle_{Q_{H, i}} \cdot y\right.$ on $\left.\partial Q_{H+\zeta(H), i}\right\}$,

where $Q_{H+\zeta(H), i}$ is the concatenation of $Q_{H, i}$ and of a crown of width $\zeta(H)$.

We then have the corresponding convergence result of Theorem 3.

THEOREM 7. In addition to $\mathrm{H} 1, \mathrm{H} 2$, and $\mathrm{H} 3$, let us assume that $p \geq 2$, that $W_{\epsilon}(x, \cdot)$ is continuously differentiable for almost all $x \in \Omega$ and $a_{\epsilon}(\cdot, 0)=\frac{\partial \bar{W}_{\epsilon}}{\partial \xi}(\cdot, 0)$ is bounded, and that $W_{\epsilon}(x, \cdot)$ satisfies the monotonicity and continuity properties (2.7) and (2.6). Then, denoting by $u_{\epsilon}$ the unique minimizer of $I_{\epsilon}$ on $W^{1, p}(\Omega)+B C$, we have

$$
\lim _{\eta \sim H \rightarrow 0} \lim _{\epsilon \rightarrow 0}\left\|\nabla u_{\epsilon}-\sum_{i=1}^{I_{H}} \nabla v_{\eta, \epsilon, w i n}^{H, i} 1_{Q_{H, i}}\right\|_{L^{p}(\Omega)}=0 .
$$

Proof of Theorem 7. The convergence of the numerical correctors is a direct consequence of Theorem 4 and [17, Thm. 2].

The proof in [17, sect. 2.4] is based on two arguments: the strong convergence of $u_{\eta, \epsilon}$ to $u_{\text {hom }}$ in $W^{1, p}(\Omega)$ and a passage from local estimates on $Q_{H, i}$ to a global estimate on $\Omega$. The first argument holds for the "windowed" method due to Theorem 4 . The local estimates are now obtained on $Q_{H+\zeta(H), i}$ and also imply a global estimate on $\Omega$ since $\lim _{H \rightarrow 0} \frac{\zeta(H)}{H}=0$. All the details of [17, sect. 2.4] adapt straightforwardly to the present case.

REMARK 8. In (4.7), one can replace the Dirichlet boundary conditions by periodic boundary conditions.

REMARK 9. A corollary of Theorem 7 shows that the family $\left\{v_{\eta, \epsilon}^{\text {win, } i}\right\}$ associated with Definition 6 and formulation (4.5) also form a corrector, which completes the convergence result of the nonconforming Petrov-Galerkin formulation of the MsFEM in the monotone case.

5. Conclusion. In numerical homogenization, the choice of the boundary conditions for the problem at the microscale in order to speed up the convergence of the numerical homogenization process is a difficult issue. It has been discussed a lot in the literature, e.g., in [23] for the community of applied mathematics and [25] for the community of mechanics. An alternative issue is given by windowing, whose aim is precisely to minimize the effect of the boundary conditions of the microscale problem. In the classical periodic and stochastic cases, windowing has proven to give better theoretical and numerical results, independently of the boundary conditions used. In the present work, we have extended the convergence results of [17] to the case of windowing. This has allowed us to prove the convergence of advanced numerical methods such as the HMM with windowing and the nonconforming Petrov-Galerkin formulation of the MsFEM in a general setting. To sum up, numerical homogenization methods with windowing indeed converge. In addition, windowing may improve the convergence of the numerical methods in two ways. Concerning the approximation of the homogenized energy, windowing does not improve the convergence rate in general but may improve the prefactor. For the numerical corrector, however, both the convergence rate and the prefactor may be improved. 


\section{REFERENCES}

[1] G. Allaire, Shape Optimization by the Homogenization Method, Appl. Math. Sci. 146, Springer-Verlag, New York, 2000.

[2] G. Allaire And R. BrizzI, A multiscale finite element method for numerical homogenization, Multiscale Model. Simul., 4 (2005), pp. 790-812.

[3] A. Bensoussan, J. L. Lions, And G. Papanicolaou, Boundary Layer Analysis in Homogenization of Diffusion Equations with Dirichlet Conditions in the Half Space, Wiley, New York, Chichester, Brisbane, 1976.

[4] A. Braides, Г-Convergence for Beginners, Oxford Lecture Ser. Math. Appl. 22, Oxford University Press, Oxford, UK, 2002.

[5] A. Braides and A. Defranceschi, Homogenization of Multiple Integrals, Oxford Lecture Ser. Math. Appl. 12, Clarendon Press, Oxford University Press, New York, 1998.

6] G. Dal Maso, An Introduction to $\Gamma$-Convergence, Birkhäuser Boston, Boston, MA, 1993.

[7] W. E, B. Engquist, X. Li, W. Ren, And E. Vanden-Eijnden, Heterogeneous multiscale methods: A review, Commun. Comput. Phys., 2 (2007), pp. 367-450.

[8] W. E, P. B. Ming, And P. W. Zhang, Analysis of the heterogeneous multiscale method for elliptic homogenization problems, J. Amer. Math. Soc., 18 (2005), pp. 121-156.

[9] Y. R. Efendiev, T. Y. Hou, ANd V. Ginting, Multiscale finite element methods for nonlinear problems and their applications, Commun. Math. Sci., 2 (2004), pp. 553-589.

[10] Y. R. Efendiev, T. Y. Hou, AND X.-H. Wu, Convergence of a nonconforming multiscale finite element method, SIAM J. Numer. Anal., 37 (2000), pp. 888-910.

[11] Y. Efendiev ANd A. PANKov, Numerical homogenization of monotone elliptic operators, Multiscale Model. Simul., 2 (2003), pp. 62-79.

[12] Y. EfEndiev AND A. PANKov, Numerical homogenization and correctors for nonlinear elliptic equations, SIAM J. Appl. Math., 65 (2004), pp. 43-68.

[13] Y. EFENDIEV AND A. PANKOV, Numerical homogenization of nonlinear random parabolic operators, Multiscale Model. Simul., 2 (2004), pp. 237-268.

[14] FreEFEM, http://www.freefem.org/.

[15] M. Giaquinta, Multiple Integrals in the Calculus of Variations and Nonlinear Elliptic Systems, Ann. of Math. Stud. 105, Princeton University Press, Princeton, NJ, 1983.

[16] M. Giaquinta and E. Giusti, On the regularity of minimizers of variational integrals, Acta Math., 148 (1982), pp. 31-46.

[17] A. Gloria, An analytical framework for the numerical homogenization of monotone elliptic operators and quasiconvex energies, Multiscale Model. Simul., 5 (2006), pp. 996-1043.

[18] A. GloRIA, A direct approach to numerical homogenization in finite elasticity, Netw. Heterog. Media, 1 (2006), pp. 109-141.

[19] T. Y. Hou And X. H. Wu, A multiscale finite element method for elliptic problems in composite materials and porous media, J. Comput. Phys., 134 (1997), pp. 169-189.

[20] T. Y. Hou, X. H. WU, AND Z. Q. CAI, Convergence of a multiscale finite element method for elliptic problems with rapidly oscillating coefficients, Math. Comp., 68 (1999), pp. 913-943.

[21] T. Y. Hou, X. H. Wu, AND Y. Zhang, Removing the cell resonance error in the multiscale finite element method via a Petrov-Galerkin formulation, Commun. Math. Sci., 2 (2004), pp. $185-205$.

[22] N. Meyers and A. Elcrat, Some results on regularity for solutions of nonlinear elliptic systems and quasiregular functions, Duke Math. J., 42 (1975), pp. 121-136.

[23] P. B. Ming And X. Y. Yue, Numerical methods for multiscale elliptic problems, J. Comput. Phys., 214 (2006), pp. 421-445.

[24] F. Murat and L. Tartar, $H$-convergence, in Topics in the Mathematical Modelling of Composites Materials, Progr. Nonlinear Differential Equations Appl. 31, A. V. Cherkaev and R. V. Kohn, eds., Birkhäuser Boston, Boston, MA, 1997, pp. 21-43.

[25] M. Ostoja-StaRZEWSKI, Material spatial randomness-from statistical to representative volume element, Prob. Eng. Mech., 21 (2006), pp. 112-132.

[26] G. Sangalli, Capturing small scales in elliptic problems using a residual-free bubbles finite element method, Multiscale Model. Simul., 1 (2003), pp. 485-503.

[27] G. Savaré, Regularity results for elliptic equations in Lipschitz domains, J. Funct. Anal., 152 (1998), pp. 176-201.

Copyright (c) by SIAM. Unauthorized reproduction of this article is prohibited. 